\title{
Review Article \\ Role of NEK2A in Human Cancer and Its Therapeutic Potentials
}

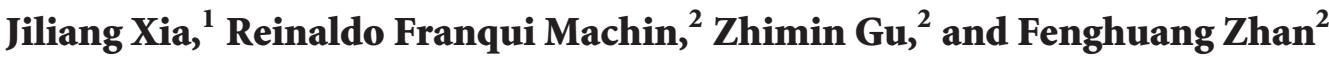 \\ ${ }^{1}$ Cancer Research Institute, Southern Medical University, Guangzhou, Guangdong 510515, China \\ ${ }^{2}$ Department of Internal Medicine, University of Iowa, Carver College of Medicine, Iowa City, IA 52242, USA \\ Correspondence should be addressed to Fenghuang Zhan; fenghuang-zhan@uiowa.edu
}

Received 19 September 2014; Accepted 14 November 2014

Academic Editor: Stefan Knapp

Copyright (C) 2015 Jiliang Xia et al. This is an open access article distributed under the Creative Commons Attribution License, which permits unrestricted use, distribution, and reproduction in any medium, provided the original work is properly cited.

\begin{abstract}
Chromosome instability (CIN) has been identified as a common feature of most human cancers. A number of centrosomal kinases are thought to cause CIN in cancer cells. Part of those centrosomal kinases exhibit elevated expression in a wide variety of tumours and cancer cell lines. Additionally, critical roles in many aspects of cancer cell growth, proliferation, metastasis, and drug resistance have been assigned to some of these centrosomal kinases, such as polo-like kinase 1 (PLk1) and Aurora-A kinase. Recent studies from our group and others revealed that a centrosomal kinase, Never in Mitosis (NIMA) Related Kinase 2A (NEK2A), is frequently upregulated in multiple types of human cancers. Uncontrolled activity of NEK2A activates several oncogenic pathways and ABC transporters, thereby leading to CIN, cancer cell proliferation, metastasis, and enhanced drug resistance. In this paper, we highlight recent findings on the aberrant expression and functional significance of NEK2A in human cancers and emphasize their significance for therapeutic potentials.
\end{abstract}

\section{Introduction}

Cancer cells tend to show some degree of genetic instability. It is now clear that high genetic change or instability plays a major role in cancer development [1]. Genetic instability can trigger tumorigenesis mainly through the activation of oncogenes and/or the inactivation of tumor suppressor genes. Chromosome instability (CIN), a phenotype characterized by a high rate of gain and/or loss of whole or large portions of chromosomes at each cell division, has been implicated in the initiation of genetic instability [2]. CIN generates a disparity in chromosome number (aneuploidy) and an enhanced rate of loss of heterozygosity, which is frequently seen in cancer cells [3-5]. Theodor Boveri observed abnormal chromosome quantities in cancer cells as early as a century ago [6]. However it was only in the recent years that CIN has been positively correlated with tumorigenesis, cancer progression, and therapeutic resistance [3-5].

Former studies have indicated that defects in cell division, telomere stability, and the DNA damage response all contribute to CIN in cancer [7]. Numerous cell division related proteins, which are highly expressed in multiple cancers, are involved in the initiation of CIN in cancer cells $[8,9]$. Centrosomal kinases are important regulators of cell division. Uncontrolled activity of centrosomal kinases can lead to spindle abnormalities, centrosome fragmentation, premature centriole splitting, multiple nucleuses, supernumerary centrosomes, and chromosome segregation errors. All those abnormal phenotype are important risk factors for CIN, indicating that overexpression of centrosomal kinases might drive tumor progression by promoting CIN $[10,11]$. Studies from our group and others have demonstrated that elevated Never in Mitosis (NIMA) Related Kinase 2A (NEK2A), a member of the NIMA-related serine/threonine kinase family and a core component of centrosome, results in CIN in cancer cells $[12,13]$. Importantly, our previous studies indicated that high expression of NEK2A is associated with poor survival in various cancers [12]. In recent years, a larger number of studies focused on the roles of NEK2A in tumorigenesis, cancer progression, and drug resistance have been published. In view of previous studies, we speculated that NEK2A may be a novel potential biomarker for diagnosis and a possible therapeutic target for human cancers. 


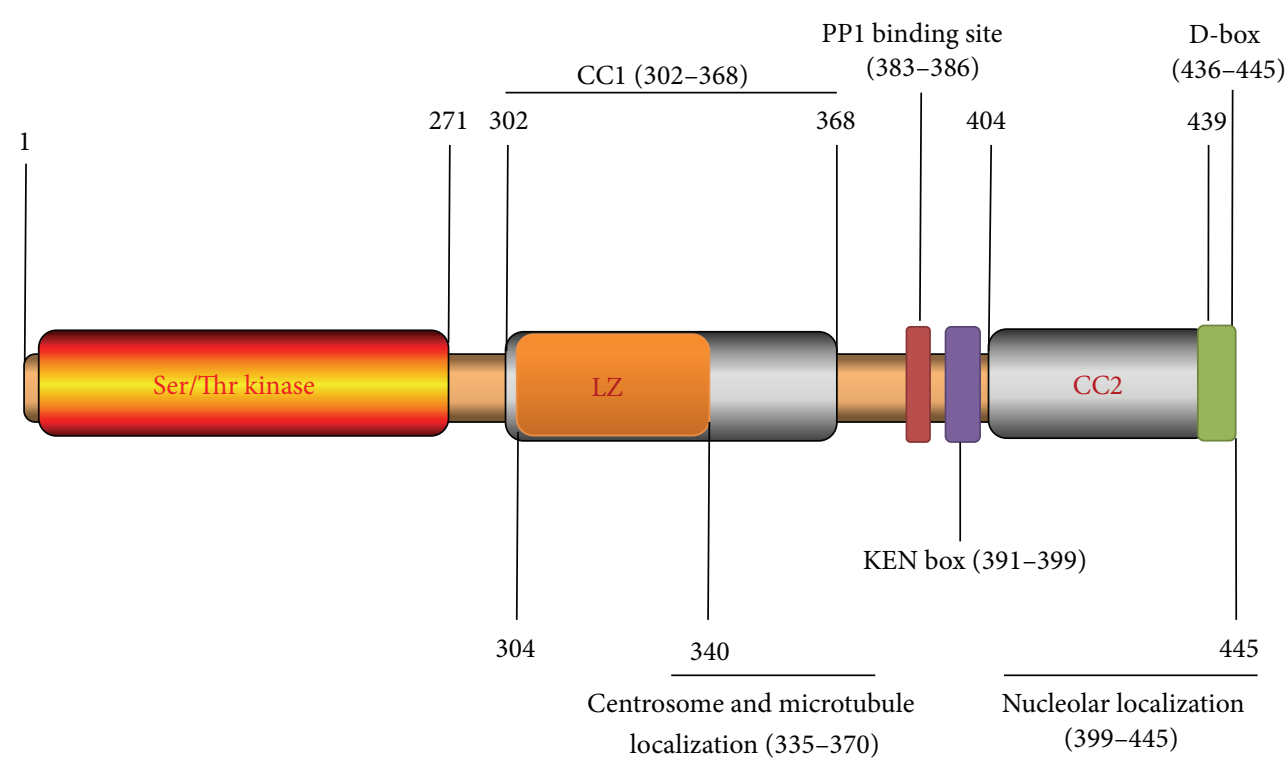

FIGURE 1: NEK2A protein structure. The relative positions of the catalytic domain (serine/threonine kinase), leucine zipper (LZ), coiled coil (CC), PP1 binding site, centrosome localization microtubule site, nucleolar localization, KEN-box, and D-box are indicated. Numbers above and below the structures indicate amino acid positions.

\section{Basic Biology of NEK2A and Validated Functions of NEK2A in Normal Cells}

The NEK2 gene in humans is located in chromosome 1 and it is comprised of 8 exons. There are three isoforms that result from the alternate splicing of this gene, termed NEK2A, $\mathrm{NEK} 2 \mathrm{~B}$, and NEK2C. NEK2A is the most studied isoform and it is a cell cycle-regulated kinase structurally related to the mitotic regulator NIMA of Aspergillus nidulans, being $47 \%$ identical within the catalytic domains [14]. NEK2A is also $31 \%$ structurally identical to Aurora-A, a human serine/threonine kinase involved in proper cell division [15]. Other structural studies demonstrated that human NEK2A is a 445 amino acid $(48 \mathrm{kDa})$ protein comprising an $\mathrm{N}$-terminal kinase domain and a $\mathrm{C}$-terminal noncatalytic regulatory domain [15]. The NEK2A N-terminal kinase domain has all the motifs typical of a serine/threonine kinase. The $\mathrm{C}$ terminal region possesses multiple regulatory motifs, which regulate the activity, location, and stability of NEK2A. These include leucine zipper (LZ), coiled coil (CC), centrosome, microtubule and nucleolar localization sites, PP1 binding site (KVHF), and APC binding site KEN-box and extended cyclin A-type destruction box (D-box) (Figure 1) [15].

Subcellular localization analysis shows that NEK2A resides in both the nucleus and cytoplasm throughout the cell cycle [16]. More detailed localization studies on the cytoplasmic NEK2A concurred to show that it is a core component of the centrosome [17]. In addition, NEK2A has been detected at nucleoli in interphase cells, on condensed chromatin in meiotic and mitotic cells, and at the kinetochores and midbody of dividing cells [16-20]. Western blot analysis demonstrates that NEK2A displays a cell cycledependent expression pattern, being low in G1, increasing through S and G2 to reach peak in late G2/M, and decreasing upon entry into mitosis $[14,21]$. Several studies have shown that a key mechanism that maintains NEK2A suppressed during $M$ phase is the ubiquitin proteasomal system (UPS) [22]. NEK2A degradation through the UPS depends on direct binding of NEK2A to the Anaphase Promoting Complex (APC/C) via two C-terminal motifs including the D-box and the KEN-box [22, 23]. This interaction leads to the ubiquitination of NEK2A and its degradation by the $26 \mathrm{~S}$ proteasome. No protein, to our knowledge, has yet been identified to stabilize NEK2A through deubiquitination; however this could also represent another aspect of NEK2A regulation. Posttranslational modifications are not the only mechanism that keeps NEK2A regulated in a cell cycle-dependent manner. Negative transcriptional regulators, like E2F4, and the epigenetic modulators, p107 and p130, negatively affect NEK2A levels directly and indirectly, respectively [24].

Similar to its expression pattern, the activity of NEK2A is cell cycle-regulated, with maximum activity in $S$ and G2 phases and low activity upon mitotic entry. NEK2A dimerization via the leucine zipper motif is essential for full activation, both in vitro and in vivo, most likely as a result of its promoting of transautophosphorylation [25]. This was shown by deleting the leucine zipper motif, which prevented the transautophosphorylation of NEK2A and reduced NEK2A activity. Many possible autophosphorylation sites of NEK2A were first identified by mass spectrometry in both the $\mathrm{N}$-terminal catalytic domain and C-terminal regulatory domain [15]. Some of these have been confirmed with in vitro kinase assays and their physiological relevance with various cell lines. Of the most important autophosphorylation sites described thus far are T175 and T179, localized in the kinase domain, which allow activation of NEK2A [15]. Other autophosphorylation sites outside the kinase domain have been described, some in the KEN-box and others in the coiled 
TABLE 1: NEK2A interaction proteins and their functions.

\begin{tabular}{|c|c|c|c|}
\hline NEK2A interaction protein & Detection method & Function & $\begin{array}{c}\text { Reference } \\
\text { number }\end{array}$ \\
\hline $\mathrm{APC} / \mathrm{C}$ & Co-IP & NEK2A degradation & {$[23]$} \\
\hline PP1 & Yeast two-hybrid, Co-IP & NEK2A dephosphorylation & {$[26,27]$} \\
\hline C-Napl & Yeast two-hybrid & Centrosome separation & {$[28]$} \\
\hline Rootletin & Yeast two-hybrid & Centrosome separation & {$[29]$} \\
\hline NLP2 & Yeast two-hybrid & Microtubule organization & {$[33]$} \\
\hline Numatrin & Co-IP, pull-down & Centrosome integrity and dynamics & {$[37]$} \\
\hline HMGA2 & Co-IP, pull-down & Chromatin condensation & {$[38]$} \\
\hline $\mathrm{HEC1}$ & Co-IP & Spindle assembly checkpoint, chromosome separation & {$[42]$} \\
\hline MAD1 & Yeast two-hybrid, Co-IP & Spindle assembly checkpoint, chromosome separation & {$[40]$} \\
\hline TRF1 & Yeast two-hybrid, pull-down & Chromosome separation & {$[41]$} \\
\hline MAD2 & Co-IP & Spindle assembly checkpoint, chromosome separation & {$[12]$} \\
\hline SGO1 & Pull-down, Co-IP & Chromosome congression & {$[43]$} \\
\hline
\end{tabular}

coil region, suggesting a role in kinase regulation and dimerization, respectively [15]. More biochemical studies must be done to understand the role of these phospho-sites. NEK2A can be negatively regulated through dephosphorylation by Protein Phosphatase 1 (PP1) that directly binds to a KVHF sequence within the C-terminal of NEK2A protein $[26,27]$. As expected, overexpression of PP1 suppresses NEK2A kinase activity, while depletion of PP1 by small interfering RNA showed increased NEK2A activity.

The subcellular localization, cell cycle-dependent expression, and activity together suggest that NEK2A may play an important role in cell division. Previous studies have demonstrated that some cell division related proteins interact with NEK2A (Table 1). Transfection of active, but not inactive NEK2A, exhibited a premature separation of centrosomes in the cell cycle, while depletion of NEK2A interferes with centrosome separation in G2 cells [17]. Subsequent studies further suggested that NEK2A induces centrosome separation by phosphorylating centrosome cohesion proteins CNap1 and Rootletin [28-30]. Besides centrosome separation, NEK2A also regulates microtubule organization through phosphorylation of ninein-like protein 2 (NLP2), resulting in its removal from the centrosome at the time of mitotic entry [31-35]. NEK2A can also help recruit numatrin to the centrosome through its kinase activity. Previously considered as noncentrosomal, recent data has surfaced that numatrin's recruitment to the centrosome protects against centrosome hyperamplification and genomic instability $[36,37]$.

Apart from its many functions in the centrosome, subcellular localization studies have found a fraction of endogenous NEK2A at condensed chromatin, particularly in cells undergoing meiosis. According to previous studies, NEK2A is activated by the MAPK pathway; it then phosphorylates an architectural chromatin protein, HMGA2. This phosphorylation decreases the affinity of HMGA2 for DNA and might drive its release from the chromatin, thereby promoting chromatin condensation $[38,39]$. NEK2A has also been reported to regulate chromosome separation by modulating the spindle assembly checkpoint (SAC). NEK2A achieves this through direct interaction or phosphorylation of mitotic arrest deficient-like 2 (MAD2), mitotic arrest deficient-like 1 (MAD1), telomere repeat binding protein (TRF1), and highly expressed in cancer 1 (HEC1) [12, 4042]. Some studies show that NEK2A regulates the alignment of chromosomes to the spindle (chromosome congression) through phosphorylating SGO1 at Ser14 and Ser507 [43]. Expression of nonphosphorylatable mutant SGO1 perturbed chromosome congression and resulted in a dramatic increase in microtubule attachment errors, including syntelic and monotelic attachments. In addition to participating in cell division, NEK2A was identified as a novel mRNA splicing factor kinase. NEK2A colocalizes in splicing speckles with SRSF1 and SRSF2, important splicing factors, and has been shown to phosphorylate the former [44]. Overexpression of NEK2A induces phosphorylation of endogenous SR proteins, a kind of proteins containing a protein domain with long repeats of serine and arginine amino acid residues, and affects the splicing activity of SRSF1 toward reporter minigenes and endogenous targets, independently of SRPK1. Conversely, knockdown of NEK2A, like that of SRSF1, induces expression of proapoptotic variants from SRSF1-target genes and sensitizes cells to apoptosis.

Although much progress has been made in our understanding of NEK2A in the past decades, several setbacks have slowed the progress in the study of this kinase. One of these is the lack of isoform-specific antibodies. Because of the similarity between NEK2A, NEK2B, and NEK2C, antibodies for each one are not available, making functional studies of endogenous NEK2A somewhat troublesome. Another problem involving the study of NEK2A is the toxicity of the wild type active protein in bacteria [66]. This does not allow the production of bulk NEK2A for crystallography experiments. The recent data uncovered by crystallography is based on unphosphorylatable mutants [15]. Another issue 
with NEK2A research is the lack of mouse models. The documented functional research of NEK2A is thus restricted to in vitro studies so far. To better characterize the in vivo role of NEK2A, mouse models of NEK2A are highly demanded. Our group has generated a NEK2A transgenic model, in which expression of NEK2A is turned on upon breeding with Cre mice in a tissue specific manner. As NEK2A is highly expressed in B cell lymphoma and multiple myeloma $[12,67]$, the NEK2A transgenic mice were bred with CD19 promoter driving cre to overexpress NEK2A in B cell lineage. Instead of developing B cell malignancies, these mice had altered B-cell development by increasing immature B-cells in the bone marrow and decreasing B-1 B-cells in peritoneal cavity. Furthermore, transgenic expression of NEK2A induced formation of spontaneous germinal centers and exhibits enhanced T-cell dependent immune response (unpublished data). All these provided the novel evidence of NEK2A's function in vivo. In addition, we are also developing NEK2A knockout mice using a gene trap strategy to better explore NEK2A's role in pathophysiological conditions.

\section{Aberrant Expression of NEK2A in Human Cancers}

Protein kinases that regulate the centrosome cycle are often aberrantly controlled in tumor cells. Changes in their expression can lead to CIN and aneuploidy, potentially triggering tumorigenesis. Increased expression of NEK2A has been reported in various cancer cells (Table 1). Reports implicating high expression of NEK2A in human cancer first appeared following microarray analysis of mRNA abundance in cancer cell line derived from Ewing tumors (ETs) (Table 2) [45]. Subsequent RT-PCR or Western blot analysis showed that multiple types of human cancer cell lines, including cholangiocarcinoma [46], testicular seminomas [47], human breast cancer [48-50], cervical cancer [50], prostate cancer [50], and colorectal Cancer $[53,54]$, expressed higher NEK2A in the level of mRNA or protein than normal human fibroblast cells. Consistently, analysis by Western blot, real-time PCR, DNA microarray, and immunohistochemistry indicated that increased NEK2A is found in various cancer tissues, such as human testicular seminomas [47], human breast carcinoma $[49,51,52]$, colorectal cancer $[53,54]$, malignant peripheral nerve sheath tumors [55], nonsmall cell lung cancer [56], renal cell carcinoma [57], and pancreatic ductal adenocarcinoma [58]. Our previous gene expression profiling (GEP) analysis showed that NEK2A expression was significantly upregulated in several types of human cancer samples compared to normal cells, including multiple myeloma, myeloid leukemia, breast cancer, lung adenocarcinoma, mantle cell lymphoma, mesothelioma, head and neck squamous cell carcinoma, bladder carcinoma, glioblastoma, T-cell acute lymphoblastic leukemia, colon carcinoma, hepatocellular carcinoma, melanoma, and ovarian adenocarcinoma [12].

Though we know NEK2A is highly expressed in various cancer cells, the underling mechanisms of increased NEK2A in cancer cells still remain poorly understood. Since both mRNA and protein of NEK2A are increased in cancer cells, several tumor-associated transcription factors and posttranslational modifications may be involved in the high expression of NEK2A in cancer cells. MicroRNA-128, a tumor suppressor, is thought to target NEK2A in colorectal cancer cell [68]. Colorectal cancer patients with high miR-128 expression had significantly lower NEK2A expression and lower recurrence rates than those with low miR-128 expression. Consistent with other tumor suppressor microRNAs, microRNA-128 is silenced by DNA methylation in colorectal cancer cells. A two- to threefold recovery of miR-128 expression was found after 5-aza-2-deoxycytidine (5aza-dC) treatment, a DNA-demethylating agent. Moreover, NEK2A expression levels were significantly reduced after 5aza-dC treatment. In addition to being indirectly inhibited by demethylation, NEK2A transcript levels are reduced by direct demethylation in HCT116 colon cancer cells, which is restricted to the distal region of the NEK2A promoter, but not in isogenic p53 $3^{-1-}$ cells [69]. Chromatin immunoprecipitation analysis demonstrated that p53 directly and specifically binds to the distal NEK2A promoter. Stabilization of endogenous p53 by doxorubicin or ectopic expression of p53, but not a p53 DNA-binding mutant, decreased NEK2A expression [69]. This study suggests that demethylation of the distal NEK2A promoter represses NEK2A expression in a p53dependent manner. As mentioned previously, in G1 and $M$ phase normal cells, NEK2A expression is downregulated by tumor suppressors including the retinoblastoma ( $\mathrm{Rb})$ family members p107 and p130 [24] and APC [22, 23]. Chromatinimmunoprecipitation (ChIP) assays demonstrated that the promoter of NEK2A is bound by E2F4 transcription factor in early G1 [24]. E2F4, a member of the E2F transcription factor family, interacts with Rb family members p107 and p130 and acts as a transcriptional repressor in G0 and G1 through recruitment of histone deacetylase which suppressed gene expression. In $\mathrm{p} 07^{-/-}$and $\mathrm{p} 130^{-/-}$mouse embryo fibroblasts (MEFs), the expression of NEK2A is significantly increased even in the absence of serum suggesting that tumours lacking p107 or p130 are likely to have elevated levels of NEK2A [24]. Moreover, overexpression of E7, a human papillomavirus encoded protein which represses the function of $\mathrm{Rb}$ family members, leads to increased NEK2A expression in human keratinocytes [70]. Forkhead transcription factor FOXM1 regulates the expression of many G2-specific genes including NEK2A and is essential for proper mitotic progression [71]. Overexpression of recombinant FOXM1 increases NEK2A expression; conversely, FOXM1 depletion reduces NEK2A expression. So far, very few reports about the relationship between NEK2A expression and tumor suppressors and oncoproteins in cancer cells have been published. Low expression of p130 and p107 or inactivated APC frequently occurs in the carcinogenic processes of multiple types of cancers [72, 73]. Both high expressions of FOXM1 and E7 are important risk factors for tumorigenesis [74, 75]. Thus elevated NEK2A in cancer cells may be induced by those abnormal conditions. Studies about the mechanisms of NEK2A expression regulation in cancers may contribute to clinical application of NEK2A-based anticancer therapeutics. 
TABLE 2: Aberrant expression of NEK2A in different cancers.

\begin{tabular}{|c|c|c|c|}
\hline Cancer type & NEK2A upregulation & Detect method & $\begin{array}{c}\text { Reference } \\
\text { number }\end{array}$ \\
\hline Ewing tumor & Cancer cell line & DNA microarray analysis & {$[45]$} \\
\hline Cholangiocarcinoma & Cancer cell line & RT-PCR, Western blot & {$[46]$} \\
\hline Testicular seminomas & Cancer cell line and tumor tissue & Immunohistochemistry, Western blot & {$[47]$} \\
\hline Breast cancer & Cancer cell line and tumor tissue & $\begin{array}{l}\text { RT-PCR, Western blot, } \\
\text { immunohistochemistry, and DNA } \\
\text { microarray analysis }\end{array}$ & [48-52] \\
\hline Cervical cancer & Cancer cell line & Western blot & {$[50]$} \\
\hline Prostate cancer & Cancer cell line & Western blot & {$[50]$} \\
\hline Colorectal cancer & Cancer cell line and tumor tissue & $\begin{array}{l}\text { Western blot, DNA microarray } \\
\text { analysis, and immunohistochemistry }\end{array}$ & {$[53,54]$} \\
\hline $\begin{array}{l}\text { Malignant peripheral nerve } \\
\text { sheath tumors }\end{array}$ & Tumor tissue & $\begin{array}{l}\text { DNA microarray analysis, } \\
\text { immunohistochemistry }\end{array}$ & {$[55]$} \\
\hline Lung adenocarcinoma & Tumor tissue & $\begin{array}{l}\text { DNA microarray analysis, } \\
\text { immunohistochemistry, } \\
\text { immunofluorescence }\end{array}$ & {$[12,56]$} \\
\hline Renal cell carcinoma & Tumor tissue & DNA microarray analysis & {$[57]$} \\
\hline $\begin{array}{l}\text { Pancreatic ductal } \\
\text { adenocarcinoma }\end{array}$ & Tumor tissue & $\begin{array}{l}\text { Real-time PCR, } \\
\text { immunohistochemistry }\end{array}$ & {$[58]$} \\
\hline Multiple myeloma & Cancer cell line and tumor tissue & DNA microarray analysis & {$[12]$} \\
\hline Myeloid leukemia & Tumor tissue & DNA microarray analysis & {$[12]$} \\
\hline Mantle cell lymphoma & Tumor tissue & DNA microarray analysis & {$[12]$} \\
\hline Mesothelioma & Tumor tissue & DNA microarray analysis & {$[12]$} \\
\hline $\begin{array}{l}\text { Head and neck squamous } \\
\text { cell carcinoma }\end{array}$ & Tumor tissue & DNA microarray analysis & {$[12]$} \\
\hline Bladder carcinoma & Tumor tissue & DNA microarray analysis & {$[12]$} \\
\hline Glioblastoma & Tumor tissue & DNA microarray analysis & {$[12]$} \\
\hline $\begin{array}{l}\text { T cell acute lymphoblastic } \\
\text { leukemia }\end{array}$ & Tumor tissue & DNA microarray analysis & {$[12]$} \\
\hline Hepatocellular carcinoma & Tumor tissue & DNA microarray analysis & {$[12]$} \\
\hline Melanoma & Tumor tissue & DNA microarray analysis & {$[12]$} \\
\hline Ovarian adenocarcinoma & Tumor tissue & DNA microarray analysis & {$[12]$} \\
\hline
\end{tabular}

\section{Roles of NEK2A in Tumorigenesis, Tumor Progression, and Drug Resistance}

Former studies have demonstrated that NEK2A involves various signaling in a broad range of cancers (Table 3 ).

4.1. Tumorigenesis. As discussed above, studies have implicated NEK2A in the regulation of centrosome separation, microtubule organization, chromatin condensation, SAC, and chromosome congression during cell division. Overexpression of NEK2A in cancer cells may result in premature centriole splitting, spindle abnormalities, multinucleation, centrosome amplification (CA), and chromosome segregation errors. These cellular phenotypes ultimately lead to CIN and aneuploidy, which is frequently observed in transformed cells with overexpressed NEK2A. This suggests that overexpression of NEK2A triggers tumorigenesis by promoting CIN and aneuploidy. Consistent with this idea, our previous studies show that overexpression of NEK2A in multiple myeloma cell results in CIN [12].
Several cell division proteins and signaling pathways are involved in NEK2A mediated CIN and aneuploidy. The MAPK pathway is required for maintaining chromatin condensed during the two meiotic divisions and uncontrolled activity of MAPK pathway has been implicated in CIN [76]. Previous studies show that NEK2A is phosphorylated by the MAPK effector $\mathrm{P} 0 \mathrm{R}^{\mathrm{sk} 2}$, thus placing these two proteins in the same pathway. Moreover, the induction of chromatin condensation requires the MAPK pathway and $\mathrm{P} \mathrm{R}^{\mathrm{sk} 2}$. Interestingly, inhibiting MAPK in the presence of okadaic acid prevents not only chromatin condensation, but also the activation of NEK2A [39]. So NEK2A may be involved in the MAPK induced CIN. The Hippo pathway components, MST2 and HSAV1, also have a direct interaction with NEK2A, thereby regulating its ability to localize to centrosome and phosphorylate C-Nap1 and Rootletin [59]. Polo-like kinase 1 (PLK1), a serine/threonine kinase identified as a potential drug target in cancer therapy, may also affect NEK2A activity in cancer cells, albeit indirectly. Tumors with 
TABLE 3: Signaling involved in the tumorigenic function of NEK2A.

\begin{tabular}{|c|c|c|c|}
\hline $\begin{array}{l}\text { Proteins or signaling pathways } \\
\text { interact with NEK } 2 \mathrm{~A}\end{array}$ & The relationship with NEK2A & Function & $\begin{array}{c}\text { Reference } \\
\text { number }\end{array}$ \\
\hline \multicolumn{4}{|l|}{ Upstream of NEK $2 A$} \\
\hline MAPK pathway & Phosphorylate NEK2A & Tumorigenesis & [39] \\
\hline MST2 & $\begin{array}{l}\text { Regulate NEK2A's ability to localize to centrosome and } \\
\text { phosphorylate C-Nap1 and Rootletin }\end{array}$ & Tumorigenesis & {$[59]$} \\
\hline PLK1 & $\begin{array}{l}\text { Regulate MST2-NEK2A/NEK2A- } \beta \text {-catenin-induced } \\
\text { centrosome separation and NEK2A-NIP-induced microtubule } \\
\text { organization }\end{array}$ & Tumorigenesis & {$[32,60,61]$} \\
\hline $\mathrm{CDK} 4$ & Regulate NEK2A expression & Tumorigenesis & {$[62]$} \\
\hline \multicolumn{4}{|l|}{ Downstream of NEK2A } \\
\hline CDC20 & Phosphorylated by NEK2A & Tumorigenesis & {$[63]$} \\
\hline MAD2 & Phosphorylated by NEK2A & Tumorigenesis & {$[63]$} \\
\hline HEC1 & Phosphorylated by NEK2A & Tumorigenesis & {$[42]$} \\
\hline TRF1 & Phosphorylated by NEK2A & Tumorigenesis & {$[64]$} \\
\hline $\mathrm{AKT}$ & Phosphorylated by NEK2A & $\begin{array}{l}\text { Tumor progression and } \\
\text { drug resistance }\end{array}$ & {$[12]$} \\
\hline$\beta$-Catenin & NEK2A induces nuclear accumulation of $\beta$-catenin & $\begin{array}{l}\text { Tumor progression and } \\
\text { drug resistance }\end{array}$ & {$[12]$} \\
\hline $\mathrm{ABCB} 1$ & Upregulated by NEK2A & Drug resistance & {$[12]$} \\
\hline $\mathrm{ABCC} 1$ & Upregulated by NEK2A & Drug resistance & {$[12]$} \\
\hline ABCG2 & Upregulated by NEK2A & Drug resistance & {$[12]$} \\
\hline BAD & Downregulated by NEK2A & Drug resistance & {$[12]$} \\
\hline PUMA & Downregulated by NEK2A & Drug resistance & {$[12]$} \\
\hline BCL-XL & Upregulated by NEK2A & Drug resistance & {$[12]$} \\
\hline MCL-1 & Upregulated by NEK2A & Drug resistance & {$[12]$} \\
\hline PARP & Activated in NEK2A silenced cancer cell & Drug resistance & {$[12]$} \\
\hline Caspase-3 & Activated in NEK2A silenced cancer cell & Drug resistance & {$[12]$} \\
\hline Caspase- 8 & Activated in NEK2A silenced cancer cell & Drug resistance & {$[12]$} \\
\hline Caspase-9 & Activated in NEK2A silenced cancer cell & Drug resistance & {$[12]$} \\
\hline $\mathrm{RB}$ & Activated in NEK2A silenced cancer cell & Drug resistance & [65] \\
\hline Histone H3 (p-Ser10) & Inactivated in NEK2A silenced cancer cell & Drug resistance & {$[65]$} \\
\hline
\end{tabular}

PLK1 overexpression were associated more frequently with CIN $(P<0.0001)$, DNA aneuploidy $(P=0.0007)$, and CA $(P=0.0013)$ than those without PLK1 overexpression [77]. Functional studies have demonstrated that PLK1 can phosphorylate MST2, and this happens upstream of the MST2NEK2A-induced centrosome separation [60]. The absence of PLK1 phosphorylation of MST2 promotes assembly of NEK2A-PP1 $\gamma$-MST2 complexes, in which PP1 $\gamma$ counteracts NEK2A kinase activity. In contrast, PLK1 phosphorylation of MST2 prevents PP1 $\gamma$ binding to MST2-NEK2A, allowing NEK2A activity to promote centrosome separation. In addition to regulating MST2-NEK2A-induced centrosome separation, PLK1 was shown to promote the NEK2A- $\beta$ catenin-induced centrosome separation [61] and NEK2ANIP-induced microtubule organization [32]. This suggests that PLK1 is an essential regulator of NEK2A in cancer cells. In summary, NEK2A has roles downstream of the MAPK pathway and PLK1; hence NEK2A may be involved in MAPKand PLK1-induced CIN and tumorigenesis.
Abnormal expression of SAC proteins can cause cell aneuploidy, an important factor in tumorigenesis. High expressions of cell division cycle 20 homolog (CDC20) and MAD2, key components of SAC, have been reported in various carcinomas. Previous studies have demonstrated that NEK2A can phosphorylate MAD2 and CDC20. Moreover, overexpression of NEK2A acts upon the MAD2-CDC20 complex and induces a delay in mitosis, promoting aneuploidy in cancer [63]. HEC1, a Ndc80 complex protein localized at kinetochores and highly expressed in cancer, is phosphorylated by NEK2A at 165-serine [42]. Overexpression of HEC1 in an inducible mouse model results in mitotic checkpoint hyperactivation and is sufficient to generate tumors that harbor significant levels of aneuploidy in vivo [78]. Former studies have demonstrated that the phosphorylation of HEC1 by NEK2A is essential for MAD1 and MAD2 to localize to the kinetochores, which is involved in $\mathrm{HECl}$ induced tumorigenesis. Their studies suggest that HEC1, MAD2, and CDC20 may be involved in NEK2A induced CIN in cancer cells. 
In Her2+ breast cancer cells, knockdown of NEK2A reduces $\mathrm{CA}$ and binucleation while its overexpression enhances CA [62]. Moreover, ectopic expression of NEK2A in immortalized HBL100 breast epithelial cells leads to accumulation of multinucleated cells with supernumerary centrosomes [50]. NEK2A expression is regulated by CDK4, which is a major regulator of CA in Her2+ breast cancer cells [62], suggesting that NEK2A may be a downstream target of CDK4, and is involved in CDK4 induced CA. Additionally, TRF1 was shown to be involved in NEK2A induced aneuploidy. It has been discovered that TRF1 interacts directly with and is phosphorylated by NEK2A. NEK2A overexpression in the breast cancer cell lines, MDA-MB231 and MCF7, results in CA and multinucleation, which leads to aneuploidy; however TRF1 depletion by siRNA prevents this phenomenon [64]. Moreover, when exogenous TRF1 was added back in NEK2A-overexpressed cells with no endogenous TRF1, cells had reinduced cytokinetic failure.

As summarized above, the expression and activity of NEK2A are regulated by many tumor suppressors and oncoproteins that show aberrant behavior in cancer. This, coupled with the abundant evidence on the effects of NEK2A on cell physiology, strongly suggests that NEK2A is an oncoprotein capable of being deregulated by several pathways. On the other hand, NEK2A regulates the activity of some cancerrelated proteins by interacting and phosphorylating them; hence NEK2A may be involved in the process of tumorigenesis.

4.2. Tumor Progression. Studies in multiple types of cancers have demonstrated that elevated NEK2A promotes cell proliferation, while its suppression with siRNA inhibited this proliferation and induced cell death [12, 46, 48-50]. Moreover, cancer cells overexpressing NEK2A showed a significant increase in colony formation compared with control cells $[12,48]$. In a xenograft nude mouse model, subcutaneous injection of NEK2A siRNA around the tumor nodules resulted in reduction of tumor size compared with those of control siRNA injection $[46,48]$. In a peritoneal dissemination model, NEK2A siRNA-treated mice showed statistically longer survival periods in comparison with those of the control siRNA treated mice [46]. Former studies show that NEK2A expression was positively associated with Ki-67 expression, a cell proliferation marker, in multiple myeloma, human primary breast cancer tissue, and nonsmall cell lung cancer $[12,49,56]$. In addition, NEK2A cytoplasmic expression was positively associated with cancer grade and tumor size in breast invasive ductal carcinoma (IDC) [51]. These data all point to NEK2A supporting tumor progression both in vitro and in vivo. Interestingly, Hayward et al. concluded that NEK2A upregulation appears to precede metastasis in their ductal carcinoma samples [50]. In line with this data, another group showed that colorectal cancer patients with high NEK2A mRNA showed greater lymph node metastasis, increased serosal, lymphatic, and venous invasion, and peritoneal dissemination when compared to the patients with low NEK2A mRNA [68]. Also, elevated NEK2A expression was maintained within all matched colorectal cancer metastases samples from NEK2Aoverexpressing primary tumours. This suggests that overexpression of NEK2A may also precede metastasis and/or help the cells survive the process in this cancer. To shed some insight on the mechanisms of the metastasis-inducing potential of NEK2A, a study in Drosophila by the Paroly group demonstrated that $\mathrm{dNek} 2$ cooperates with Ras and Src signaling to promote metastasis. Coexpression of dNek2 along with activated Ras and $\mathrm{Src}$ (dNek2; $\mathrm{Csk}^{-/-}$; $\mathrm{Ras}^{\mathrm{V} 12}$ cell) led to significant overgrowth of tumor cells as well as appearance of secondary tumors in the body of the larvae. In tumor cell injection assays, $\mathrm{dNek} 2 ; \mathrm{Csk}^{-/-}$; $\mathrm{Ras}^{\mathrm{V} 12}$ tumor cells were injected into the dorsal notum region of wild type (WT) adult flies, and within 10 days of injection tumor cells could be seen in various parts of the adult body. However, injection of dNek 2 cells or $\mathrm{Csk}^{-/-}$; Ras ${ }^{\mathrm{V} 12}$ cells did not result in detectable tumor populations in the other body parts [79]. This strongly suggests that metastasis induced by NEK2A works in conjunction with other pathways, like Ras. Taken together, this data indicates a pivotal role of NEK2A in tumorigenic growth and progression; however the underling mechanisms are still poorly understood.

In a previous study, we showed that the AKT inhibitor LY294002 and $\beta$-catenin shRNA decrease the NEK2A induced colony formation in multiple myeloma, suggesting that both PP1/AKT and the Wnt signaling pathway may be involved in NEK2A-induced cell proliferation [12]. Evidence of NEK2A involved in Wnt signaling has been uncovered by other groups as well. An excellent example comes from Neal et al. in colorectal cancer [54]. In this study, NEK2A overexpression was associated with lower tumour membranous $\beta$-catenin expression and higher cytoplasmic and nuclear $\beta$ catenin accumulation [54]. Our previous study also showed that overexpressions of NEK2A in multiple myeloma and lung cancer cells induce nuclear accumulation of $\beta$-catenin [12]. $\beta$-Catenin localization from the intercellular adherens junction to the cytoplasm and nucleus is characteristic of tumor metastasis; thus NEK2A may play an important role in tumor metastasis through regulating the expression and localization of $\beta$-catenin. Our preliminary data also showed that NEK2A increases $\beta$-catenin transcriptional activity and exhibits role of antisenescence through increasing phosphorylation of $\mathrm{Rb}$ (unpublished data).

4.3. Drug Resistance. Drug resistance is one of the main problems in cancer treatment. Our previous studies have implicated NEK2A in cancer cell drug resistance [12]. Multiple myeloma cells transfected to overexpress NEK2A showed only a slight decrease in their capacity to form colonies when treated with Bortezomib, doxorubicin, and Etoposide. However, control cells transfected with empty vectors showed a significant decrease in colony formation when incubated with these drugs at the same concentrations. Studies from another research group showed that both NEK2A and pololike kinase 4 (PLk4) are highly expressed in Her2-positive breast cancer cells exhibiting trastuzumab resistance [52]. NEK2A expression is upregulated in drug-resistant ovarian cancer cells as well, when compared with their sensitive 
or parental counterparts. Thus it is clear that NEK2A has a role in cancer cell drug resistance. To understand how NEK2A generates this resistant phenotype, we conducted flow cytometry in search for apoptotic cells. The results indicated that multiple myeloma cells overexpressing NEK2A showed lesser cell apoptosis after treatment with anticancer drugs than control cells without NEK2A overexpression. Consistently, shRNA-mediated NEK2A depletion overcame myeloma cell drug resistance and induced apoptosis in vitro and in a xenograft myeloma mouse model [12]. A bioinformatic analysis consisting of protein/gene-protein/gene interaction networks, annotation of biological processes, and microRNA-mRNA interaction indicated that NEK2A directly or indirectly interacts with a number of genes, proteins, and microRNAs [80]. This study also suggested NEK2A had implications in biological processes associated with drug resistance in ovarian and other types of cancer [80]. In our study, Western blot results showed that overexpression of NEK2A in cancer cells upregulated ABC transporter family members, including ABCB1 ( $\mathrm{p}$-glycoprotein, MDR1), the multidrug resistance protein $\mathrm{ABCC} 1$ (MRP1), and the breast cancer resistant protein ABCG2 [12]. Consistently, downregulation of NEK2A by shRNA decreased the expression of these ABC transporters. To corroborate that the NEK2Ainduced increase of $\mathrm{ABC}$ transporters contributes to drug resistance, a flow cytometry-based analysis was performed. This showed that cancer cells overexpressing NEK2A have a higher efflux of the hydrophilic eFluxx-ID gold fluorescent dye compared with control cells, indicating higher activity of ABC transporters in NEK2A-elevated cancer cells. Verapamil, an ABC transporter inhibitor, was able to abrogate part of the NEK2A-induced drug resistance by showing a decrease in colony formation. Our data strongly suggest that NEK2A induces drug resistance mainly through enhancing the activation of ABC transporters. Our subsequent studies further indicated that both PP1/AKT and canonical Wnt signaling were involved in NEK2A-induced activation of $\mathrm{ABC}$ transporters [12]. Inhibition of AKT or knockdown of $\beta$-catenin in NEK2A-overexpressed myeloma cells inhibits the expression of $\mathrm{ABC}$ transporters $\mathrm{ABCB} 1, \mathrm{ABCC} 1$, and ABCG2; moreover, there was a decreased efflux of the hydrophilic eFluxx-ID gold fluorescent dye in those cells. This suggests that NEK2A induction of ABC transporters involves $\mathrm{AKT}$ and $\beta$-catenin. In addition, we found that overexpression of NEK2A in cancer cells suppressed the expression of the proapoptotic genes BAD and PUMA and upregulated the expression of prosurvival genes BCL-XL and MCL-1 [12]. Depletion of NEK2A in cancer cells increased the level of cleaved PARP and activation of caspase-3, caspase-8, and caspase-9, indicating a possible role of NEK2A against the apoptosis pathway [12]. The other group also found that NEK2A knockdown in breast cancer cells induces aneuploidy, cell cycle arrest, and caspase-dependent and independent cell death. Mechanistically, NEK2A depletion in breast cancer cell increases caspase- 3 cleavage and promotes the activity of the tumor suppressor $\mathrm{Rb}$ while simultaneously reducing the activation of the cell division regulator histone $\mathrm{H} 3$ [65]. Because induction of apoptosis is one of the main mechanisms of anticancer drugs use to stimulate cell death,
NEK2A-induced antiapoptosis may explain the high cancer cell drug resistance seen when NEK2A is increased.

Many cancers avoid apoptosis and generate drug resistance after chemotherapeutic agents by activating prosurvival mechanisms like autophagy [81]. Many independent groups have shown that autophagy can antagonize apoptosis and other forms of cell death after drug treatment [80]. This is particularly important for multiple myeloma, a cancer high in NEK2A expression and elevated autophagic flux [82]. NEK2A has been shown to alter pathways like AKT and be activated by MAPK, as discussed previously. Because these two pathways are important modulators of autophagy, it is likely that NEK2A could be altering autophagy, as a means to sustain malignant cells after drug treatment. Increased autophagy by NEK2A could be a novel mechanism by which cancer cells acquire drug resistance; however, to our knowledge, no group has yet exploited this approach. The study of autophagy regulation by NEK2A could provide more insight on the currently misunderstood NEK2A-derived malignancy and also the autophagic process. We summarized oncogenic function of NEK2A in Figure 2.

\section{Therapeutic Potential of NEK2A}

The rationale for exploring the therapeutic potential of NEK2A is based on the observations described above that implicate NEK2A in various human cancers, contributing to tumorigenesis, tumour progression, and drug resistance. In recent years, several studies focused on the relationship between NEK2A and cancer clinicopathological factors. To explore the roles of NEK2A in human breast cancer progression, researchers correlated the expression of NEK2A with some of the clinicopathological factors in human breast cancer tissue. As a result, NEK2A mRNA expression was associated with certain molecular subtypes, like Estrogen Receptor (ER), Progesterone Receptor (PR), and Ki-67 immunoreactivity $(P<0.05)$ in breast ductal carcinoma in situ (DCIS) tissue; moreover, in IDC tissue, NEK2A expression was associated with histological grade, lymph node metastasis, molecular subtypes, C-erbB-2 expression, and Ki-67 expression $(P<0.05)[49]$. Breast cancer patients with high expression of NEK2A exhibited higher mortality and recurrence rate than NEK2A low expression patients. In human pancreatic cancer, overexpression of NEK2A was significantly correlated with histological differentiation $(P=$ $0.042)$, lymph node metastasis $(P=0.003)$, and tumor stage $(P=0.001)$ [58]. Pancreatic cancer patients with a high NEK2A expression also had a significantly worse overall survival than those patients with low NEK2A expression $(P=0.002)$. Likewise, nonsmall cell lung cancer patients with overexpression of NEK2A also had a poorer overall survival rate compared to those with low expression for all stages $(P=0.000)$ [56]. Colorectal cancer patients with high NEK2A expression had a significantly poorer prognosis than those with low NEK2A expression [68]. Moreover, univariate and multivariate analysis showed that NEK2A mRNA expression was an independent prognostic indicator of overall survival in patients with colorectal cancer [68]. 


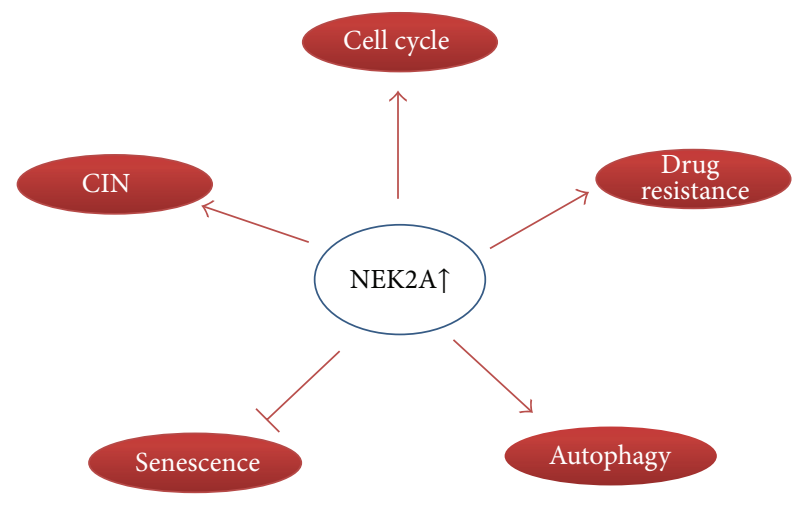

FIgURE 2: Summary of oncogenic activity of NEK2A.

In addition, our previous A Kaplan-Meier survival analysis has indicated that high expression of NEK2A is linked to poor survival in multiple myeloma [12]. The same clinical implication of high NEK2A expression is also observed in other cancers, including acute myeloid leukemia, bladder cancer, breast cancer, glioma, lung adenocarcinoma, mantle cell lymphoma, and mesothelioma [12]. Taken together, those data suggest that NEK2A is a novel potential biomarker for diagnosis and a possible therapeutic target for cancer.

Overexpressing NEK2A in cancer cells resulted in enhanced cancer progression and drug resistance, while targeting NEK2A with shRNA overcame cancer cell drug resistance and induced apoptosis. Therefore, downregulation or inactivation of NEK2A in cancer cells may contribute to cancer therapy. In recent years, based on the spatial structure of NEK2A, a number of specific NEK2A inhibitors have been developed through high-throughput screening [83-86]. A small molecular inhibitor for NEK2A and HEC1 binding 1 (INH1) has been first found to specifically disrupt the $\mathrm{HEC1/NEK2A} \mathrm{interaction} \mathrm{via} \mathrm{direct} \mathrm{HEC1} \mathrm{binding} \mathrm{thereby}$ leading to metaphase chromosome misalignment, spindle aberrancy, and eventual cell death [87]. Treatment with INH1 suppresses the proliferation of multiple human breast cancer cells in vitro. In vivo, INH1 retarded tumor growth in a nude mouse model bearing xenografts derived from the human breast cancer line MDA-MB-468, with no apparent side effects. In recent years, researchers successively developed many more effective INH, such as INH41 [88], INH154 [88], TAI-1 [89], and TAI-95 [90]. These inhibitors had $\mathrm{IC}_{50}$ in nm level and suppressed the growth of multiple types of cancer cells but had no significant growth inhibitory effects on the nontumorigenic cells [88-90]. In addition, these inhibitors not only disrupt HEC1-NEK2A protein interaction but also promote NEK2A degradation through the proteasome pathway and may act as powerful cancer therapeutic for NEK2A and $\mathrm{HECl}$ overexpressing cancers. A study examining the effect of a combination treatment using NEK2A siRNA with the chemotherapeutic agent cisplatin (CDDP) on a colorectal cancer model indicated that administration of NEK2A siRNA with CDDP results in the suppression of tumor growth compared to the single administration of NEK2A siRNA or control siRNA and CDDP [53]. Targeting NEK2A by siRNA or antisense oligonucleotides (ASOs) in breast cancer cells increased drug sensitivity. These results suggest that combination treatment using NEK2A siRNA and chemotherapeutic agents may be effective and can serve as a therapeutic option for the treatment of cancer.

\section{Conclusion}

As reviewed above, NEK2A contributes to several biological processes of the tumor cell, including proliferation, metastasis, and drug resistance. Studies from our group and others have indicated that elevated expression of NEK2A is positively correlated with molecular subtypes, tumor stage, poor prognosis, and poor overall survival rate. These studies together suggest that NEK2A may be a novel potential therapeutic target for human cancers. Because NEK2A has such a broad spectrum of roles in different cell processes, it is expected that by targeting this kinase, several tumor promoting pathways will be affected, greatly improving treatment outcome. Some research groups have already developed various NEK2A inhibitors, which have been shown to effectively suppress tumor growth in xenograft nude mouse model; thus the outlook on this field appears promising. On the other hand, because initial data has shown NEK2A can predict patient prognosis, more research on its efficiency in predicting disease stage and overall outcome is greatly encouraged, since this data can help generate a more personalized and efficient treatment for the cancer patient in the future. Overall, we anticipate that further studies will provide more convincing support for NEK2A-based therapy strategies for various cancers.

\section{Abbreviations}

CIN: Chromosome instability

PLK1: Polo-like kinase 1

NEK2A: Never in Mitosis (NIMA) Related Kinase 2A

APC/C: Anaphase Promoting Complex

PP1: $\quad$ Protein Phosphatase 1

NLP2: Ninein-like protein 2

SAC: Spindle assembly checkpoint

MAD1: Mitotic arrest deficient-like 1

MAD2: Mitotic arrest deficient-like 2

TRF1: Telomere repeat binding protein 1

HEC1: Highly expressed in cancer 1

ETs: Ewing tumors

GEP: Gene expression profiling

RB: Retinoblastoma

FOXM1: Forkhead transcription factor 1

CA: Centrosome amplification

CDC20: Cell division cycle 20 homolog

IDC: Invasive ductal carcinoma

DCIS: Ductal carcinoma in situ

ER: $\quad$ Estrogen Receptor

PR: $\quad$ Progesterone Receptor

INH1: Inhibitor for NEK2A and HEC1 binding 1. 


\section{Conflict of Interests}

The authors declare that there is no conflict of interests regarding the publication of this paper.

\section{Acknowledgments}

This work was supported by National Cancer Institute Grants R01CA152105 (to F.Z.) and P30 CA086862; the Leukemia Lymphoma Society TRP (to F.Z., 6246-11 and 6094-12); Institutional Startup Funds from the Department of Internal Medicine, Carver School of Medicine, University of Iowa (to F.Z.).

\section{References}

[1] C. Lengauer, K. W. Kinzler, and B. Vogelstein, "Genetic instabilities in human cancers," Nature, vol. 396, no. 6712, pp. 643-649, 1998.

[2] S. L. Thompson, S. F. Bakhoum, and D. A. Compton, "Mechanisms of chromosomal instability," Current Biology, vol. 20, no. 6, pp. R285-R295, 2010.

[3] M. A. Nowak, N. L. Komarova, A. Sengupta et al., "The role of chromosomal instability in tumor initiation," Proceedings of the National Academy of Sciences of the United States of America, vol. 99, no. 25, pp. 16226-16231, 2002.

[4] K. Fukasawa, "Centrosome amplification, chromosome instability and cancer development," Cancer Letters, vol. 230, no. 1, pp. 6-19, 2005.

[5] S. F. Bakhoum and D. A. Compton, "Chromosomal instability and cancer: a complex relationship with therapeutic potential," The Journal of Clinical Investigation, vol. 122, no. 4, pp. 1138-1143, 2012.

[6] T. Boveri, "Über mehrpolige mitosen als mittel zur analyse des zellkerns," Verhandlungen der Physikalisch-Medizinische Gesellschaft, vol. 35, pp. 67-90, 1902.

[7] S. M. Gollin, "Mechanisms leading to chromosomal instability," Seminars in Cancer Biology, vol. 15, no. 1, pp. 33-42, 2005.

[8] N. J. Ganem, S. A. Godinho, and D. Pellman, "A mechanism linking extra centrosomes to chromosomal instability," Nature, vol. 460, no. 7252, pp. 278-282, 2009.

[9] G. de Cárcer and M. Malumbres, "A centrosomal route for cancer genome instability," Nature Cell Biology, vol. 16, no. 6, pp. 504-506, 2014.

[10] J.-M. Schvartzman, R. Sotillo, and R. Benezra, "Mitotic chromosomal instability and cancer: mouse modelling of the human disease," Nature Reviews Cancer, vol. 10, no. 2, pp. 102-115, 2010.

[11] J. J. Li and S. A. Li, "Mitotic kinases: the key to duplication, segregation, and cytokinesis errors, chromosomal instability, and oncogenesis," Pharmacology \& Therapeutics, vol. 111, no. 3, pp. 974-984, 2006.

[12] W. Zhou, Y. Yang, J. Xia et al., "NEK2 induces drug resistance mainly through activation of efflux drug pumps and is associated with poor prognosis in myeloma and other cancers," Cancer Cell, vol. 23, no. 1, pp. 48-62, 2013.

[13] D. G. Hayward and A. M. Fry, "Nek2 kinase in chromosome instability and cancer," Cancer Letters, vol. 237, no. 2, pp. 155$166,2006$.

[14] S. J. Schultz, A. M. Fry, C. Sutterlin, T. Ried, and E. A. Nigg, "Cell cycle-dependent expression of Nek2, a novel human protein kinase related to the NIMA mitotic regulator of Aspergillus nidulans," Cell Growth and Differentiation, vol. 5, no. 6, pp. 625635, 1994.

[15] P. Rellos, F. J. Ivins, J. E. Baxter et al., "Structure and regulation of the human Nek2 centrosomal kinase," The Journal of Biological Chemistry, vol. 282, no. 9, pp. 6833-6842, 2007.

[16] H. K. Yong, Y. C. Jun, Y. Jeong, D. J. Wolgemuth, and K. Rhee, "Nek2 localizes to multiple sites in mitotic cells, suggesting its involvement in multiple cellular functions during the cell cycle," Biochemical and Biophysical Research Communications, vol. 290, no. 2, pp. 730-736, 2002.

[17] A. M. Fry, P. Meraldi, and E. A. Nigg, "A centrosomal function for the human Nek2 protein kinase, a member of the NIMA family of cell cycle regulators," The EMBO Journal, vol. 17, no. 2, pp. 470-481, 1998.

[18] K. Rhee and D. J. Wolgemuth, "The NIMA-related kinase 2, Nek2, is expressed in specific stages of the meiotic cell cycle and associates with meiotic chromosomes," Development, vol. 124, no. 11, pp. 2167-2177, 1997.

[19] T. Fujioka, Y. Takebayashi, M. Ito, and T. Uchida, "Nek2 expression and localization in porcine oocyte during maturation," Biochemical and Biophysical Research Communications, vol. 279, no. 3, pp. 799-802, 2000.

[20] R. S. Hames, R. E. Crookes, K. R. Straatman et al., "Dynamic recruitment of Nek2 kinase to the centrosome involves microtubules, PCM-1, and localized proteasomal degradation," Molecular Biology of the Cell, vol. 16, no. 4, pp. 1711-1724, 2005.

[21] A. M. Fry, S. J. Schultz, J. Bartek, and E. A. Nigg, "Substrate specificity and cell cycle regulation of the Nek2 protein kinase, a potential human homolog of the mitotic regulator NIMA of Aspergillus nidulans," The Journal of Biological Chemistry, vol. 270, no. 21, pp. 12899-12905, 1995.

[22] R. S. Hames, S. L. Wattam, H. Yamano, R. Bacchieri, and A. M. Fry, "APC/C-mediated destruction of the centrosomal kinase Nek2A occurs in early mitosis and depends upon a cyclin Atype D-box," The EMBO Journal, vol. 20, no. 24, pp. 7117-7127, 2002.

[23] G. G. Sedgwick, D. G. Hayward, B. di Fiore et al., "Mechanisms controlling the temporal degradation of Nek2A and Kif18A by the APC/C-Cdc20 complex," The EMBO Journal, vol. 32, no. 2, pp. 303-314, 2013.

[24] B. Ren, H. Cam, Y. Takahashi et al., "E2F integrates cell cycle progression with DNA repair, replication, and $G_{2} / M$ checkpoints," Genes \& Development, vol. 16, no. 2, pp. 245-256, 2002.

[25] A. M. Fry, L. Arnaud, and E. A. Nigg, "Activity of the human centrosomal kinase, Nek2, depends on an unusual leucine zipper dimerization motif," The Journal of Biological Chemistry, vol. 274, no. 23, pp. 16304-16310, 1999.

[26] N. R. Helps, X. Luo, H. M. Barker, and P. T. W. Cohen, "NIMArelated kinase 2 (Nek2), a cell-cycle-regulated protein kinase localized to centrosomes, is complexed to protein phosphatase 1," Biochemical Journal, vol. 349, no. 2, pp. 509-518, 2000.

[27] J. Mi, C. Guo, D. L. Brautigan, and J. M. Larner, "Protein phosphatase- $1 \alpha$ regulates centrosome splitting through Nek2," Cancer Research, vol. 67, no. 3, pp. 1082-1089, 2007.

[28] A. M. Fry, T. Mayor, P. Meraldi, Y. D. Stierhof, K. Tanaka, and E. A. Nigg, "C-Nap1, a novel centrosomal coiled-coil protein and candidate substrate of the cell cycle-regulated protein kinase Nek2," Journal of Cell Biology, vol. 141, no. 7, pp. 1563-1574, 1998.

[29] S. Bahe, Y.-D. Stierhof, C. J. Wilkinson, F. Leiss, and E. A. Nigg, "Rootletin forms centriole-associated filaments and functions 
in centrosome cohesion," The Journal of Cell Biology, vol. 171, no. 1, pp. 27-33, 2005.

[30] A. J. Faragher and A. M. Fry, "Nek2A kinase stimulates centrosome disjunction and is required for formation of bipolar mitotic spindles," Molecular Biology of the Cell, vol. 14, no. 7, pp. 2876-2889, 2003.

[31] R. Gräf, "DdNek2, the first non-vertebrate homologue of human Nek2, is involved in the formation of microtubuleorganizing centers," Journal of Cell Science, vol. 115, no. 9, pp. 1919-1929, 2002.

[32] J. Rapley, J. E. Baxter, J. Blot et al., "Coordinate regulation of the mother centriole component Nlp by Nek2 and Plk1 protein kinases," Molecular and Cellular Biology, vol. 25, no. 4, pp. 13091324, 2005.

[33] Y. Jeong, J. Lee, K. Kim, J. C. Yoo, and K. Rhee, "Characterization of NIP2/centrobin, a novel substrate of Nek2, and its potential role in microtubule stabilization," Journal of Cell Science, vol. 120, no. 12, pp. 2106-2116, 2007.

[34] S. Sonn, Y. Jeong, and K. Rhee, "Nip2/centrobin may be a substrate of Nek2 that is required for proper spindle assembly during mitosis in early mouse embryos," Molecular Reproduction and Development, vol. 76, no. 6, pp. 587-592, 2009.

[35] S. Sonn, G. T. Oh, and K. Rhee, "Nek2 and its substrate, centrobin/Nip2, are required for proper meiotic spindle formation of the mouse oocytes," Zygote, vol. 19, no. 1, pp. 15-20, 2011.

[36] S. Grisendi, C. Mecucci, B. Falini, and P. P. Pandolfi, "Nucleophosmin and cancer," Nature Reviews Cancer, vol. 6, no. 7, pp. 493-505, 2006.

[37] J. Yao, C. Fu, X. Ding et al., "Nek2A kinase regulates the localization of numatrin to centrosome in mitosis," FEBS Letters, vol. 575, no. 1-3, pp. 112-118, 2004.

[38] S. di Agostino, M. Fedele, P. Chieffi et al., "Phosphorylation of high-mobility group protein A2 by Nek2 kinase during the first meiotic division in mouse spermatocytes," Molecular Biology of the Cell, vol. 15, no. 3, pp. 1224-1232, 2004.

[39] S. Di Agostino, P. Rossi, R. Geremia, and C. Sette, “The MAPK pathway triggers activation of Nek2 during chromosome condensation in mouse spermatocytes," Development, vol. 129, no. 7, pp. 1715-1727, 2002.

[40] Y. Lou, J. Yao, A. Zereshki et al., "NEK2A interacts with MAD1 and possibly functions as a novel integrator of the spindle checkpoint signaling," The Journal of Biological Chemistry, vol. 279, no. 19, pp. 20049-20057, 2004.

[41] G. Prime and D. Markie, "The telomere repeat binding protein Trf1 interacts with the spindle checkpoint protein Mad1 and Nek2 mitotic kinase," Cell Cycle, vol. 4, no. 1, pp. 121-124, 2005.

[42] R. Wei, B. Ngo, G. Wu, and W. H. Lee, "Phosphorylation of the Ndc80 complex protein, HEC1, by Nek2 kinase modulates chromosome alignment and signaling of the spindle assembly checkpoint," Molecular Biology of the Cell, vol. 22, no. 19, pp. 3584-3594, 2011.

[43] G. Fu, X. Ding, K. Yuan et al., "Phosphorylation of human Sgo1 by NEK2A is essential for chromosome congression in mitosis," Cell Research, vol. 17, no. 7, pp. 608-618, 2007.

[44] C. Naro, F. Barbagallo, P. Chieffi, C. F. Bourgeois, M. P. Paronetto, and C. Sette, "The centrosomal kinase NEK2 is a novel splicing factor kinase involved in cell survival," Nucleic Acids Research, vol. 42, no. 5, pp. 3218-3227, 2014.

[45] D. H. Wai, K.-L. Schaefer, A. Schramm et al., "Expression analysis of pediatric solid tumor cell lines using oligonucleotide microarrays," International Journal of Oncology, vol. 20, no. 3, pp. 441-451, 2002.
[46] T. Kokuryo, T. Senga, Y. Yokoyama, M. Nagino, Y. Nimura, and M. Hamaguchi, "Nek2 as an effective target for inhibition of tumorigenic growth and peritoneal dissemination of cholangiocarcinoma," Cancer Research, vol. 67, no. 20, pp. 9637-9642, 2007.

[47] F. Barbagallo, M. P. Paronetto, R. Franco et al., "Increased expression and nuclear localization of the centrosomal kinase Nek2 in human testicular seminomas," The Journal of Pathology, vol. 217, no. 3, pp. 431-441, 2009.

[48] N. Tsunoda, T. Kokuryo, K. Oda et al., "Nek2 as a novel molecular target for the treatment of breast carcinoma," Cancer Science, vol. 100, no. 1, pp. 111-116, 2009.

[49] S. Wang, W. Li, N. Liu et al., "Nek2A contributes to tumorigenic growth and possibly functions as potential therapeutic target for human breast cancer," Journal of Cellular Biochemistry, vol. 113, no. 6, pp. 1904-1914, 2012.

[50] D. G. Hayward, R. B. Clarke, A. J. Faragher, M. R. Pillai, I. M. Hagan, and A. M. Fry, "The centrosomal kinase Nek2 displays elevated levels of protein expression in human breast cancer," Cancer Research, vol. 64, no. 20, pp. 7370-7376, 2004.

[51] S. Wang, W. Li, S. Lv et al., "Abnormal expression of Nek2 and $\beta$ catenin in breast carcinoma: clinicopathological correlations," Histopathology, vol. 59, no. 4, pp. 631-642, 2011.

[52] M. Marina and H. Saavedra, "Nek2 and Plk4: prognostic markers, drivers of breast tumorigenesis and drug resistance," Frontiers in Bioscience, vol. 19, pp. 352-365, 2014.

[53] K. Suzuki, T. Kokuryo, T. Senga, Y. Yokoyama, M. Nagino, and M. Hamaguchi, "Novel combination treatment for colorectal cancer using Nek2 siRNA and cisplatin," Cancer Science, vol. 101, no. 5, pp. 1163-1169, 2010.

[54] C. P. Neal, A. M. Fry, C. Moreman et al., "Overexpression of the Nek2 kinase in colorectal cancer correlates with beta-catenin relocalization and shortened cancer-specific survival," Journal of Surgical Oncology, vol. 110, no. 7, pp. 828-838, 2014.

[55] T. P. Stricker, K. J. Henriksen, J. H. Tonsgard, A. G. Montag, T. N. Krausz, and P. Pytel, "Expression profiling of 519 kinase genes in matched malignant peripheral nerve sheath tumor/plexiform neurofibroma samples is discriminatory and identifies mitotic regulators $B U B 1 B, P B K$ and $N E K 2$ as overexpressed with transformation," Modern Pathology, vol. 26, no. 7, pp. 930-943, 2013.

[56] X. Zhong, X. Guan, Q. Dong et al., "Examining Nek2 as a better proliferation marker in non-small cell lung cancer prognosis," Tumor Biology, vol. 35, no. 7, pp. 7155-7162, 2014.

[57] Y. Cheng, M. Hong, and B. Cheng, "Identified differently expressed genes in renal cell carcinoma by using multiple microarray datasets running head: differently expressed genes in renal cell carcinoma," European Review for Medical and Pharmacological Sciences, vol. 18, no. 7, pp. 1033-1040, 2014.

[58] Z. Ning, A. Wang, J. Liang et al., "Abnormal expression of Nek2 in pancreatic ductal adenocarcinoma: a novel marker for prognosis," International Journal of Clinical and Experimental Pathology, vol. 7, no. 5, pp. 2462-2469, 2014.

[59] B. R. Mardin, C. Lange, J. E. Baxter et al., "Components of the Hippo pathway cooperate with Nek2 kinase to regulate centrosome disjunction," Nature Cell Biology, vol. 12, no. 12, pp. 1166-1176, 2010.

[60] B. R. Mardin, F. G. Agircan, C. Lange, and E. Schiebel, "Plk1 controls the Nek2A-PP1 $\gamma$ antagonism in centrosome disjunction," Current Biology, vol. 21, no. 13, pp. 1145-1151, 2011.

[61] B. C. Mbom, K. A. Siemers, M. A. Ostrowski, W. J. Nelson, and A. I. M. Barth, "Nek2 phosphorylates and stabilizes $\beta$-catenin 
at mitotic centrosomes downstream of Plk1," Molecular Biology of the Cell, vol. 25, no. 7, pp. 977-991, 2014.

[62] M. K. H. Pitner and H. I. Saavedra, "Cdk4 and nek2 signal binucleation and centrosome amplification in a her2+ breast cancer model," PLoS ONE, vol. 8, no. 6, Article ID e65971, 2013.

[63] Q. Liu, Y. Hirohashi, X. Du, M. I. Greene, and Q. Wang, "Nek2 targets the mitotic checkpoint proteins Mad2 and Cdc20: a mechanism for aneuploidy in cancer," Experimental and Molecular Pathology, vol. 88, no. 2, pp. 225-233, 2010.

[64] J. Lee and L. Gollahon, "Mitotic perturbations induced by Nek2 overexpression require interaction with TRF1 in breast cancer cells," Cell Cycle, vol. 12, no. 23, pp. 3599-3614, 2013.

[65] P. Cappello, H. Blaser, C. Gorrini et al., "Role of Nek2 on centrosome duplication and aneuploidy in breast cancer cells," Oncogene, vol. 33, no. 18, pp. 2375-2384, 2014.

[66] A. M. Fry and E. A. Nigg, "Characterization of mammalian NIMA-related kinases," Methods in Enzymology, vol. 283, pp. 270-282, 1997.

[67] U. Andreasson, M. Dictor, M. Jerkeman et al., "Identification of molecular targets associated with transformed diffuse large B cell lymphoma using highly purified tumor cells," The American Journal of Hematology, vol. 84, no. 12, pp. 803-808, 2009.

[68] Y. Takahashi, T. Iwaya, G. Sawada et al., "Up-regulation of NEK2 by MicroRNA-128 methylation is associated with poor prognosis in colorectal cancer," Annals of Surgical Oncology, vol. 21, no. 1, pp. 205-212, 2014.

[69] N. H. Nabilsi, D. J. Ryder, A. C. Peraza-Penton, R. Poudyal, D. S. Loose, and M. P. Kladde, "Local depletion of DNA methylation identifies a repressive p53 regulatory region in the NEK2 promoter," The Journal of Biological Chemistry, vol. 288, no. 50, pp. 35940-35951, 2013.

[70] D. Patel, A. Incassati, N. Wang, and D. J. McCance, "Human papillomavirus type 16 E6 and E7 cause polyploidy in human keratinocytes and up-regulation of G 2-M-phase proteins," Cancer Research, vol. 64, no. 4, pp. 1299-1306, 2004.

[71] D. R. Wonsey and M. T. Follettie, "Loss of the forkhead transcription factor FoxM1 causes centrosome amplification and mitotic catastrophe," Cancer Research, vol. 65, no. 12, pp. 5181-5189, 2005.

[72] J.-H. Dannenberg, L. Schuijff, M. Dekker, M. Van Der Valk, and H. Te Riele, "Tissue-specific tumor suppressor activity of retinoblastoma gene homologs p107 and p130," Genes and Development, vol. 18, no. 23, pp. 2952-2962, 2004.

[73] R. Fodde, R. Smits, and H. Clevers, "APC, signal transduction and genetic instability in colorectal cancer," Nature Reviews Cancer, vol. 1, no. 1, pp. 55-67, 2001.

[74] A. Ahmad, Z. Wang, D. Kong et al., "FoxM1 down-regulation leads to inhibition of proliferation, migration and invasion of breast cancer cells through the modulation of extra-cellular matrix degrading factors," Breast Cancer Research and Treatment, vol. 122, no. 2, pp. 337-346, 2010.

[75] M. Narisawa-Saito and T. Kiyono, "Basic mechanisms of highrisk human papillomavirus-induced carcinogenesis: roles of E6 and E7 proteins," Cancer Science, vol. 98, no. 10, pp. 1505-1511, 2007.

[76] H. I. Saavedra, K. Fukasawa, C. W. Conn, and P. J. Stambrook, "MAPK mediates RAS-induced chromosome instability," The Journal of Biological Chemistry, vol. 274, no. 53, pp. 3808338090, 1999.

[77] Y. Yamamoto, H. Matsuyama, S. Kawauchi et al., "Overexpression of polo-like kinase 1 (PLK1) and chromosomal instability in bladder cancer," Oncology, vol. 70, no. 3, pp. 231-237, 2006.
[78] E. Diaz-Rodríguez, R. Sotillo, J.-M. Schvartzman, and R. Benezra, "Hecl overexpression hyperactivates the mitotic checkpoint and induces tumor formation in vivo," Proceedings of the National Academy of Sciences of the United States of America, vol. 105, no. 43, pp. 16719-16724, 2008.

[79] T. K. Das, D. Dana, S. S. Paroly et al., "Centrosomal kinase Nek2 cooperates with oncogenic pathways to promote metastasis," Oncogenesis, vol. 2, article e69, 2013.

[80] X. Liu, Y. Gao, Y. Lu, J. Zhang, L. Li, and F. Yin, "Upregulation of NEK2 is associated with drug resistance in ovarian cancer," Oncology Reports, vol. 31, no. 2, pp. 745-754, 2014.

[81] R. Mathew, V. Karantza-Wadsworth, and E. White, "Role of autophagy in cancer," Nature Reviews Cancer, vol. 7, no. 12, pp. 961-967, 2007.

[82] B. Hoang, A. Benavides, Y. Shi, P. Frost, and A. Lichtenstein, "Effect of autophagy on multiple myeloma cell viability," Molecular Cancer Therapeutics, vol. 8, no. 7, pp. 1974-1984, 2009.

[83] D. G. Hayward, Y. Newbatt, L. Pickard et al., "Identification by high-throughput screening of viridin analogs as biochemical and cell-based inhibitors of the cell cycle-regulated Nek2 kinase," Journal of Biomolecular Screening, vol. 15, no. 8, pp. 918927, 2010.

[84] P. Innocenti, K.-M. J. Cheung, S. Solanki et al., "Design of potent and selective hybrid inhibitors of the mitotic kinase nek2: structure-activity relationship, structural biology, and cellular activity," Journal of Medicinal Chemistry, vol. 55, no. 7, pp. 32283241, 2012.

[85] X.-L. Qui, G. Li, G. Wu et al., "Synthesis and biological evaluation of a series of novel inhibitor of Nek2/Hecl analogues," Journal of Medicinal Chemistry, vol. 52, no. 6, pp. 1757-1767, 2009.

[86] H. Lebraud, C. R. Coxon, V. S. Archard et al., "Model system for irreversible inhibition of Nek2: thiol addition to ethynylpurines and related substituted heterocycles," Organic and Biomolecular Chemistry, vol. 12, no. 1, pp. 141-148, 2014.

[87] G. Wu, X.-L. Qiu, L. Zhou et al., "Small molecule targeting the Hec1/Nek2 mitotic pathway suppresses tumor cell growth in culture and in animal," Cancer Research, vol. 68, no. 20, pp. 8393-8399, 2008.

[88] C.-M. Hu, J. Zhu, X. E. Guo et al., "Novel small molecules disrupting Hec1/Nek2 interaction ablate tumor progression by triggering Nek2 degradation through a death-trap mechanism," Oncogene, 2014.

[89] L. Y. Huang, Y.-S. Lee, J.-J. Huang et al., "Characterization of the biological activity of a potent small molecule Hecl inhibitor TAI-1," Journal of Experimental and Clinical Cancer Research, vol. 33, article 6, 2014.

[90] L. Y. Huang, C. C. Chang, Y. S. Lee et al., "Inhibition of Hecl as a novel approach for treatment of primary liver cancer," Cancer Chemotherapy and Pharmacology, vol. 74, no. 3, pp. 511-520, 2014. 


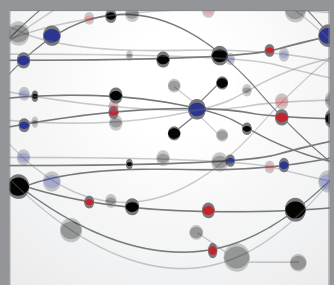

The Scientific World Journal
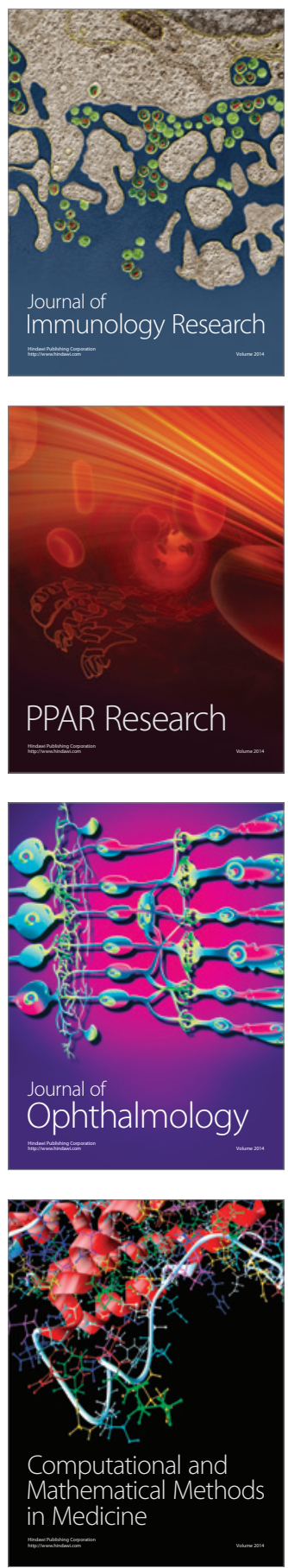

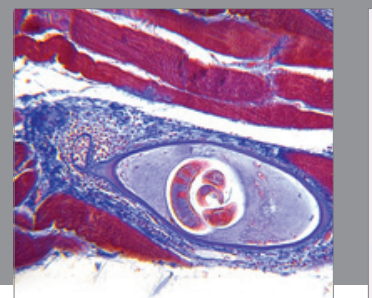

Gastroenterology

Research and Practice
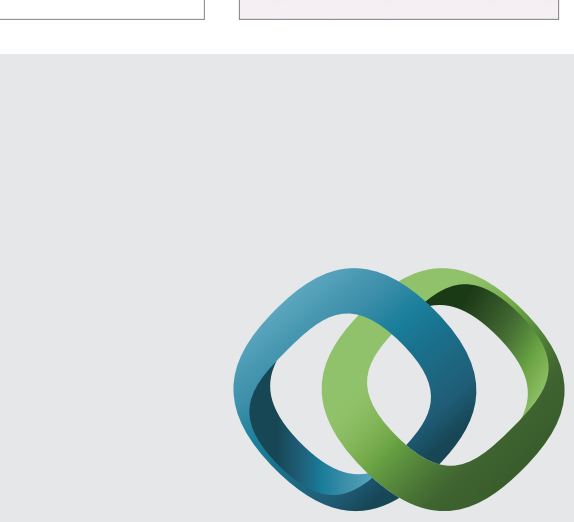

\section{Hindawi}

Submit your manuscripts at

http://www.hindawi.com
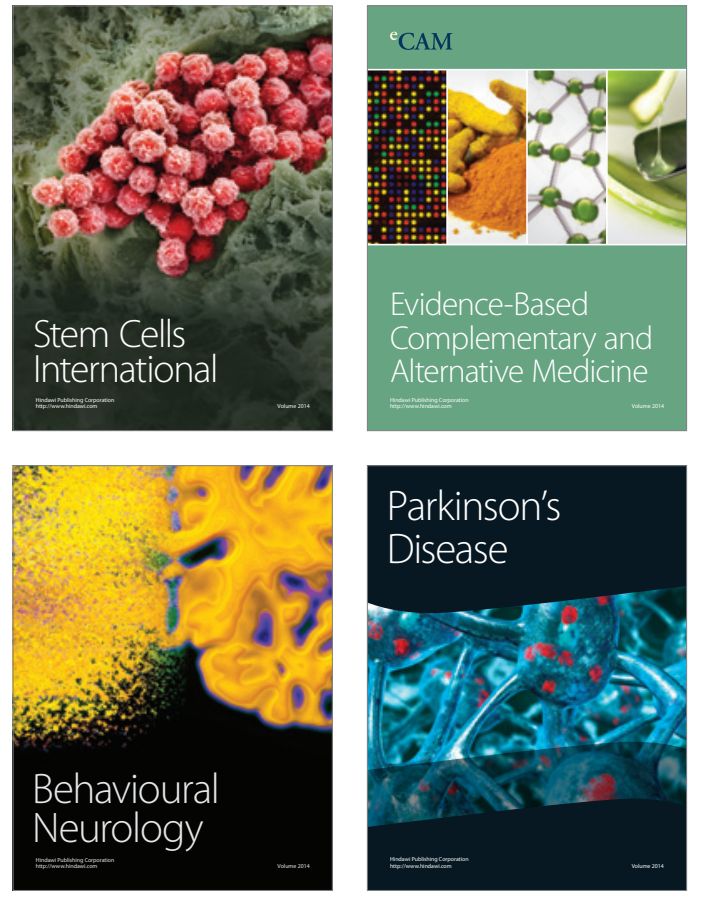
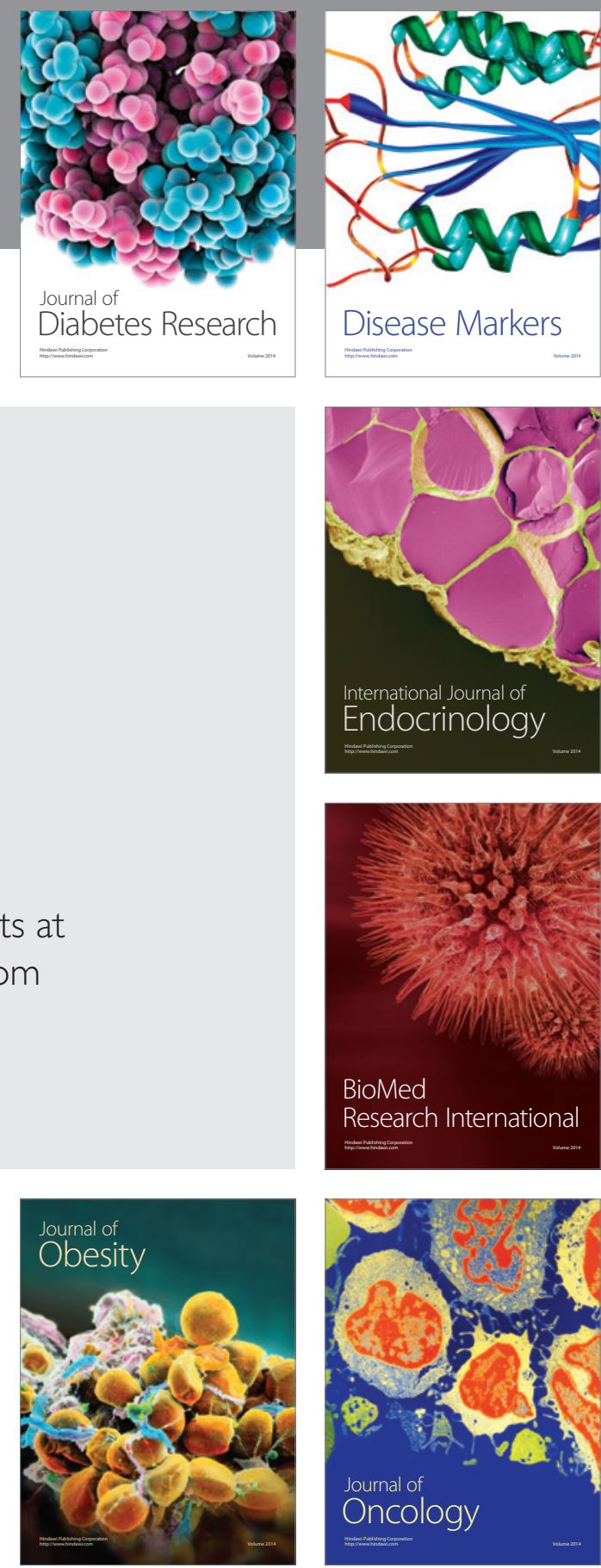

Disease Markers
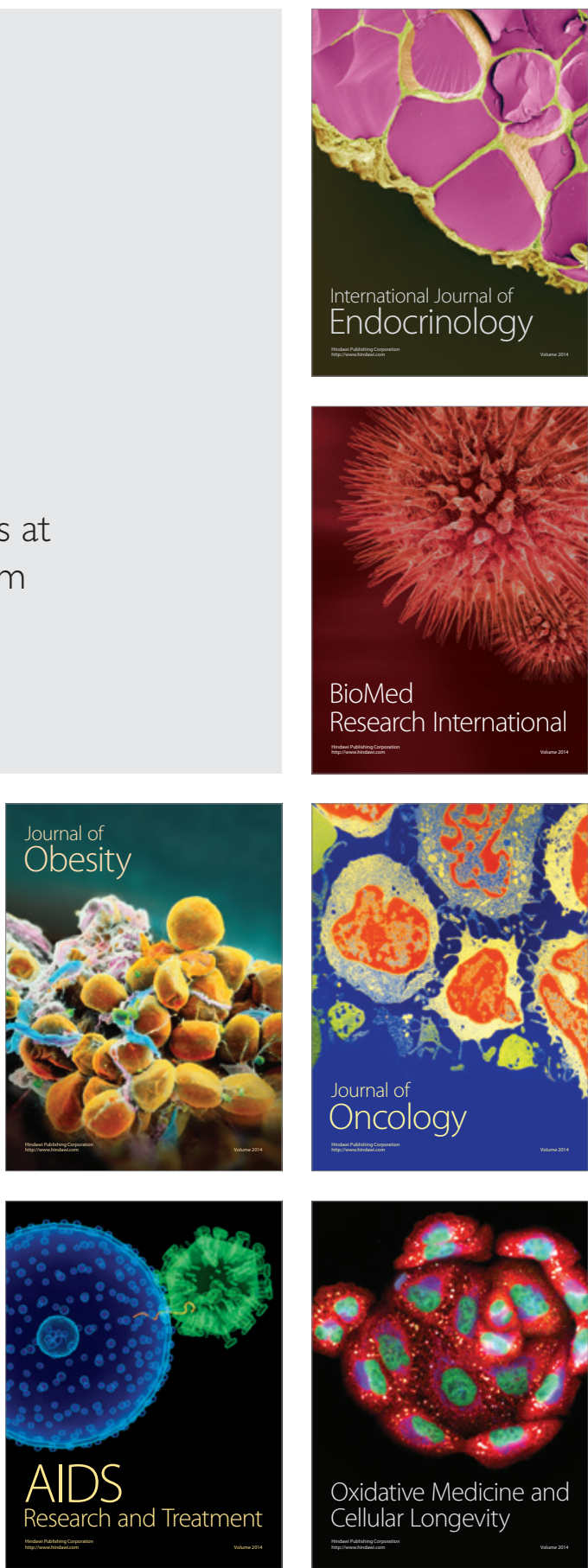